\title{
Contents of Lactate and Intracellular Oxygen Metabolism at Neutrophils in Patients with Acute Coronary Syndrome
}

\author{
Andrey Eu. Kratnov \\ Department of Therapy of the Paediatric Faculty of Yaroslavl State Medical Academy, \\ Yaroslavl, Russia
}

Correspondence should be addressed to: Andrey Eu. Kratnov; kratnov@mail.ru

Received date: 29 November 2013; Accepted date: 13 February 2014; Published date: 3 December 2015

Academic Editor: Joydip Mukherjee

Copyright @ 2015. Andrey Eu. Kratnov. Distributed under Creative Commons CC-BY 4.0

\begin{abstract}
The contents of lactate and intracellular oxygen metabolism at neutrophils in 56 patients with acute coronary syndrome have been investigated. In the process of weighting acute coronary syndrome, the growth of formation of active oxygen radicals at neutrophils (growth of parameters the test of nitroblue tetrazolium reduction and myeloperoxidase activity) which was not compensated by sufficient increase in intracellular activity of enzymes of antioxidative system protection (catalase activity), and was accompanied by accumulation the lactate in neutrophils.
\end{abstract}

Keywords: acute coronary syndrome, neutrophils, reactive forms of oxygen, lactate.

\section{Introduction}

Increased quantity of neutrophils in peripheral blood, which play an important role in reperfusion damage of myocardium, attracts attention among prognostic markers of risk cardiac death in these decades [10, 13]. In experiment it has been shown that, activation of formation, the oxygen radicals at neutrophils at myocardial ischemia/reperfusion was accompanied by accumulation malonylaldehyde and lactate dehydrogenase in plasma of blood and associated with reduction of survival rate [4]. The aim of this study was to investigate the intracellular oxygen metabolism, and contents of lactate at neutrophils in patients with acute coronary syndrome (ACS).

\section{Methodology}

56 people with ACS (mean age $69.3 \pm 11.6$ years) among which 24 patients $(42.9 \%)$ with unstable angina, 7 (12.5\%) patients with nonQ-wave myocardial infarction, and 25 (44.6\%) patients with Q-wave myocardial infarction have been examined. Among the observed patients with ACS, the quantity of men and women authentically did not differ. Control group has consisted from 40 (41.7\%) patients (mean age $41.2 \pm 12.4$ years) without coronary heart disease. In order to exclude coronary heart disease in patients of control group, electrocardiography (ECG) was performed, along with bicycle ergometry, Holter ECG monitoring, and echocardiography. The criteria of exception of this research: smoking, type 2 diabetes

Cite this Article as: Andrey Eu. Kratnov (2015), " Contents of Lactate and Intracellular Oxygen Metabolism at Neutrophils in Patients with Acute Coronary Syndrome", JMED Research, Vol. 2015 
mellitus, obesity, bronchial asthma, cancer, and dipsomania.

The material for this study was peripheral blood analysis which was performed at admission of patients to the cardiology intensive care with the consent of the Ethical Committee. Neutrophils were isolated from heparinised blood in Ficoll-Verographin double density gradient 1.077 and 1.092 $\mathrm{g} / \mathrm{cm}^{3}$. The second interface cells contained $95 \%$ of neutrophils. The number of neutrophils in the cell suspension was counted in a cell with Goryaev vivo staining with methylene blue in 3\% acetic acid (Turk dye) to determine viable cells. Viability of phagocytes, estimated by trypan test was more than $90 \%$. In order to achieve a concentration of $5 \times 10^{6}$, neutrophils in $1 \mathrm{ml}$ of cell suspension was diluted with medium 199.

The test of spontaneous and stimulated nitro blue tetrasolium reduction (NBT-test) was performed by means of quantitative spectrophotometric method using $0.2 \%$ nitro blue tetrazolium in phosphate buffer, fixing neutrophils with methanol and dissolving in a mixture of reduced diformazan potassium hydroxide and dimethylsulfoxide 3:5 volume mixture [8]. For evaluation of neutrophils $\mathrm{C}_{3}{ }^{-}$ receptor activity, phytohemagglutinin from kidney beans (Phaseolus vulgaris) was used.

Myeloperoxidase activity in neutrophils was studied using a $0.04 \%$ solution ortho phenylenediaminein phosphate buffer $\mathrm{pH}$ 5.0, with the addition of $0.33 \%$ hydrogen peroxide solution at a ratio of 20:1 by volume. The reaction was stopped after 10 minutes of $10 \%$ sulphuric acid solution. Photometry was carried out at $\lambda=492 \mathrm{nM}$ [12].

The determination of catalase activity in neutrophils was based on the ability of hydrogen peroxide to form salts with molybdenum stable colored complex. The reaction was run by adding $0.03 \%$ hydrogen peroxide solution and was stopped after 10 minutes, adding a $4 \%$ solution of ammonium molybdate. In control test, instead of the neutrophils distilled water was brought. The color intensity was measured at $\lambda=410 \mathrm{nM}$ against a control sample of distilled water [11].

The definition contents of lactate at neutrophils was based on the ability of hydrogen peroxide formed at transformation of lactate in pyruvate under action lactate oxidase, to cooperate with $n$-chlorphenol and 4-aminoantipirini with formation painted product which spectrophotometrically was determined [15].

The data were processed by parametric and nonparametric methods using Statistical 8.0 software (Stat Soft, Inc.). The data of research were presented as the mean and standard deviation $(M \pm S D)$. The differences between the groups were considered significant at $p<$ 0.05 .

\section{Results}

The development of ACS was accompanied by an increase in the contents of C-reactive protein $(36 \pm 36.2>2.5 \pm 2.3 \mathrm{mg} / \mathrm{l}, p=$ 0.00004 ) and the quantity of neutrophils (67 $\pm 10.2>58.3 \pm 7,9 \%, p=0.00002)$ in peripheral blood (Figure 1). In patients with ACS, in comparison with a group of patients without coronary heart disease, authentically higher parameters stimulated

NBT-test $(112.4 \pm 26.6>100.1 \pm 29.1 \mathrm{nM}, p=$ $0.02)$, the increase of activity myeloperoxidase $(17.6 \pm 8.2>14.3 \pm 4.2$ Sigma units, $p=0.02$ ), and reduction of activity catalase $(507.8 \pm 366.1<730.2 \pm$ $276.9 \mathrm{mCat} / \mathrm{l}, p=0.002$ ) at neutrophils were observed. The activation of neutrophils on a background of growth level of circulating immune complexes in blood (117.8 $\pm 82.6>$ $96.2 \pm 32.2$ units of optical density) was observed. It was accompanied by an authentic increase in the contents of lactate at neutrophils $(1.9 \pm 0.9>1.2 \pm 0.3 \mathrm{mMol} / \mathrm{l}, p=$ 0.002 ) in patients with ACS. 


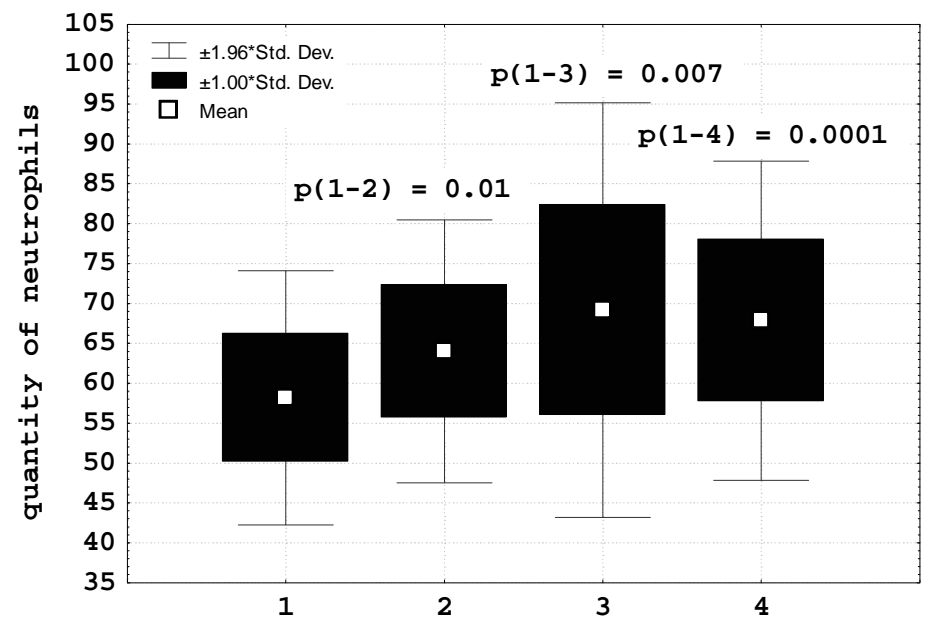

(1) - patients without coronary heart disease;

(2) - patients with unstable angina;

(3) - patients with non-Q-wave myocardial infarction;

(4) - patients with Q-wave myocardial infarction.

Figure 1: Neutrophilia in patients with acute coronary syndrome.

At studying a condition of intracellular metabolism of neutrophils depending on the form (unstable angina, non-Q-wave myocardial infarction) of ACS, it is revealed that, the greatest parameters spontaneous
$(104.5 \pm 19.1>83.6 \pm 18.8$ and $96.2 \pm 23 \mathrm{nM})$ and stimulated NBT-test $(120.5 \pm 27.5>$ $103.5 \pm 28$ and $107.6 \pm 24.2 \mathrm{nM}$ ) in patients with Q-wave myocardial infarction were observed (Figure 2).

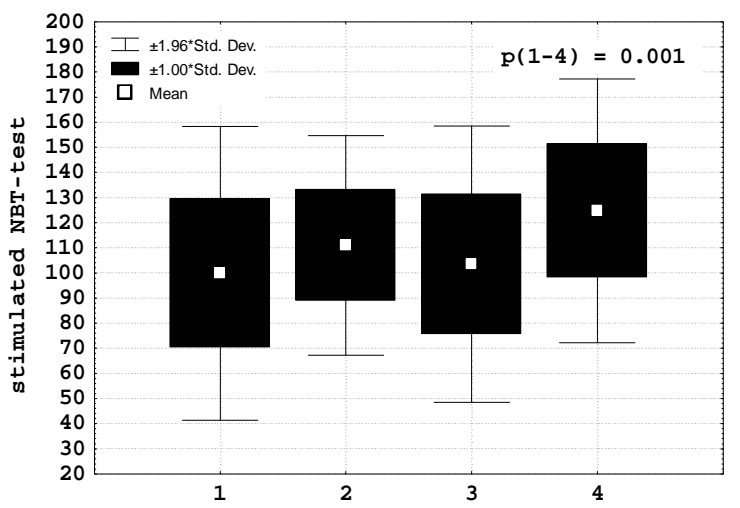

(1) - patients without coronary heart disease;

(2) - patients with unstable angina;

(3) - patients with non-Q-wave myocardial infarction;

(4) - patients with Q-wave myocardial infarction.

Figure 2: Stimulated NBT-test in patients with acute coronary syndrome. 
IBIMA Publishing

JMED Research

http://www.ibimapublishing.com/journals/JMED/jmed.html

Vol. 2015 (2015), Article ID 581306, 6 pages

DOI: $10.5171 / 2015.581306$

Growth the formation of superoxide anionradical at neutrophils in patients with Q-wave

accompanied by the growth of the and non-Q-wave myocardial infarction was

intracellular contents of lactate $(2.1 \pm 1.1$ and $1.9 \pm 0.7>1.5 \pm 0.4 \mathrm{mMol} / \mathrm{l}$ ) (Figure 3)

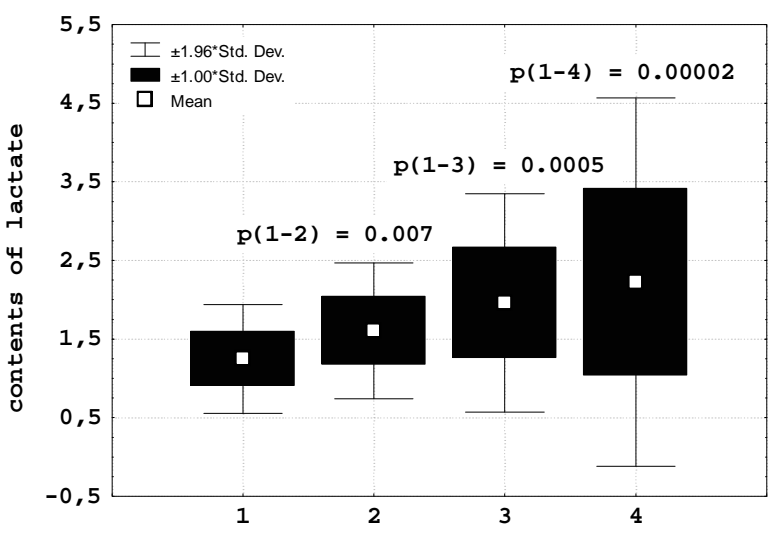

(1) - patients without coronary heart disease;

(2) - patients with unstable angina;

(3) - patients with non-Q-wave myocardial infarction;

(4) - patients with Q-wave myocardial infarction.

Figure 3: Contents of lactate at neutrophils in patients with acute coronary syndrome.

on a background of increase in activity myeloperoxidase $(20.3 \pm 9$ and $20.3 \pm 6.5>$ $13.9 \pm 6.4$ Sigma units) (Figure 4) and catalase $(611.2 \pm 443.7$ and $456.6 \pm 268>$ $442.4 \pm 317.2 \mathrm{mCat} / \mathrm{l})$ which parameters, however, did not reach values of patients without coronary heart disease. Thus, on a measure of weighting ACS, the growth of formation oxygen radicals at neutrophils was not compensated by the sufficient increase, the activity of intracellular system of antioxidative protection, and was accompanied by accumulation of lactate.

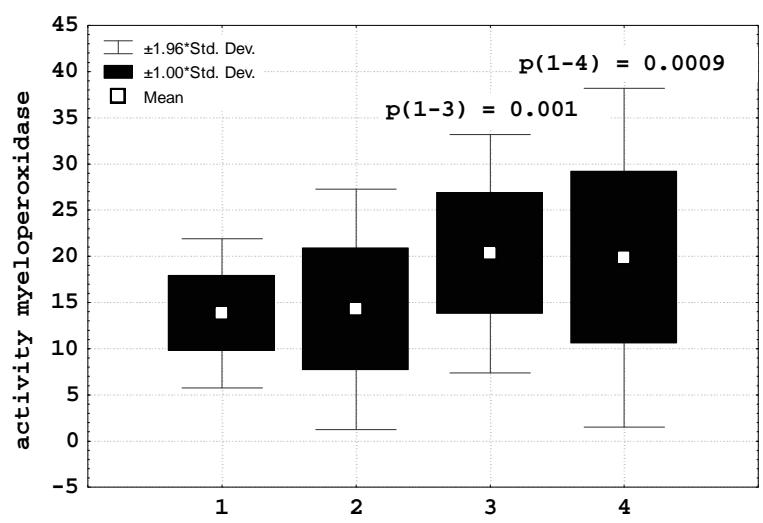

(1) - patients without coronary heart disease;

(2) - patients with unstable angina;

(3) - patients with non-Q-wave myocardial infarction;

(4) - patients with Q-wave myocardial infarction.

Figure 4: Activity myeloperoxidase at neutrophils in patients with acute coronary syndrome. 


\section{Discussion}

It is known, that the rule of development ACS is a spontaneous thrombolysis which accompanying the development of reperfusion syndrome [3]. Takes for granted, that the activated neutrophils are the important source of oxygen radicals at reperfused myocardium. Inflammatory reaction in ischemic myocardium characterized the early phase of infiltration of neutrophils (24-48 hours) with the subsequent infiltration of monocytes in myocardium was revealed. Neutrophils collapse inside myocardium through 2 mines after reperfusion that is accompanied by an increase of the concentration lysosomal enzymes at coronary sine in comparison with peripheral blood $[14,16]$.

It is known, that lactate dehydrogenase is biochemical marker of myocardial damage [1]. The experimental data, which allowing making the assumption, that lactate-induced myocardial ischemic injury is a consequence of increased intracellular lactate leading to inhibition of glycolysis presumably via an increased NADH/NAD ratio, are received [5]. In the given research in patients with ACS, the activation of NADPH oxidase at neutrophils on the data of NBT-test was accompanied by the growth of the contents intracellular lactate. It is possible to believe, that in conditions of acute hypoxia in ASC at neutrophils which for respiratory processes, use mainly glycolysis instead of mitochondrial oxidizing phosphorylation [2], the increase to formation of lactate, the capable to promoting development of intracellular acidosis. There are assumptions that the high concentration $\mathrm{H}^{+}$strengthens action of oxygen factors at phagocyte.

Also, lactate working as osmotic attractant is capable to break integrity of plasmatic membrane and to initiate autodestruction of cells [9]. In the given research, in patients with ACS significant direct correlation between parameters lactate and activity myeloperoxidase at neutrophils, came to light $(\mathrm{R}=0.62, p=0.000001)$. Believe that antineutrophil interventions can effectively prevent the increase in oxygen radical concentration during reperfusion [6]. It is revealed, that the inhibition of $\mathrm{Na}^{+} / \mathrm{H}^{+}$ exchange with using 5-methyl-N-isobutyl amiloride shows a protective effect against neutrophil-induced reperfusion injury [7].

\section{Conclusions}

The increase in formation of active forms of oxygen at neutrophils in acute coronary syndrome associates with the growth of the intracellular contents of lactate.

\section{References}

1. Bhayana, V. and Henderson, AR. (1995), 'Biochemical markers of myocardial damage,' Clin. Biochem., 28(1), 1-29.

2. Borregaard, N. and Herlin, T. (1982), 'Energy metabolism of human neutrophils during phagocytosis,' J. Clin. Invest., (70), 550-557.

3. Bulkely, BH. and Hutchins, GM. (1977), 'Myocardial consequences of coronary artery bypass graft surgery. The paradox of necrosis in areas of revascularization,' Circulation, (56), 906-913.

4. Campo, GM, Squadrito, F., Altavilla, D., Squadrito, G., Avenoso, A., Canale, P., Ioculano, M., Sperandeo, A. and Caputi, AP. (1996), 'Protection of ischemic and reperfused rat myocardium by the nonglucocorticoid 21aminosteroid U-74389G, a new inhibitor of lipid peroxidation,' J. Pharmacol. Exp. Ther., 277 (1), 333-340.

5. Cross, HR., Clarke, K., Opie, LH. and Radda, GK. (1995), 'Is lactate-induced myocardial ischaemic injury mediated by decreased $\mathrm{pH}$ or increased intracellular lactate?' J. Mol. Cell. Cardiol., 27(7), 1369-81.

6. Duilio, C., Ambrosio, G., Kuppusamy, P., DiPaula, A., Becker, LC. and Zweier, JL. (2001), 'Neutrophils are primary source of $\mathrm{O} 2$ radicals during reperfusion after prolonged myocardial ischemia,' Am. J. Physiol. Heart Circ. Physiol., 280(6), H2649-H2657.

7. Faes, FC., Sawa, Y., Ichikawa, H., Shimazaki, Y., Ohashi, T., Fukuda, H., 
Shirakura, R. and Matsuda, H. (1995), 'Inhibition of $\mathrm{Na}^{+} / \mathrm{H}^{+}$exchanger attenuates neutrophil-mediated reperfusion injury,' Ann. Thorac. Surg., 60(2), 377-381.

8. Gentle, TA. and Thompson, RA. (1990), Neutrophil function tests in clinical immunology, Clinical immunology, A practical approach, Gooi, HG. and Chapel, H. (ed), Oxford University Press, New York.

9. Hart, PD. (1982), Lysosome fusion responses of macrophages to infection: behavior and significance, New York.

10. Kyne, L, Hausdorff, JM., Knight, E., Dukas, L., Azhar, G. and Wei, JY. (2000), 'Neutrophilia and congestive heart failure after acute myocardial infarction,' Am. Heart J., (139), 94-100.

11. Mamontova, NS, Beloborodova, EI. and Tucalova, LI. (1994), 'Activity of catalase at chronic dipsomania,' Klin. Lab. Diagn., (1), 27 28.

12. Saidov, MZ. and Pinegin, BV. (1991), 'Spectrophotometric analysis of myeloperoxidase activity in phagocytes,' Laboratornoe delo, (3), 56-59.
13. Sato, H., Jordan, JE, Zhao, ZQ., Sarvotham, SS. and VintenJohansen, J. (1997), 'Gradual reperfusion reduces infarct size and endothelial injury but augments neutrophil accumulation,' Ann. Thorac. Surg., (64), 10991107.

14. Simpson, PJ, Fantone, JC, Mickelson, JK, Gallagher, KP. and Lucchesi, BR. (1998), 'Identification of a time window for therapy to reduce experimental canine myocardial injury: suppression of neutrophil activity during 72 hours of reperfusion,' Circ. Res., (63), 1070-1079.

15. Sommerhof, CP., Nadel, JA., Basbaum, CB. and Caughey, GH. (1990), 'Neutrophil elastase and cathepsin $G$ stimulated secretion from cultured bovine airway gland serous cell,' J. Clin. Invest., (85), 682-689.

16. Williams, FM, Collins, PD, Tannieze-Zeller M. and Williams TJ. (1990), "The relationship between neutrophils and increased microvascular permeability in a model of myocardial ishemia and reperfusion in the rabbit,' J. Pharmacol., (100), 7829-7834. 